Proceedings

\title{
Towards High-Power Printed Textile Supercapacitors ${ }^{\dagger}$
}

\author{
Paulo Luís ${ }^{1, *}$, Jagdeep Sagu ${ }^{1}$, Darren Southee ${ }^{2}$, Upul Wijayantha ${ }^{1}$ and George Weaver ${ }^{1}$ \\ 1 Department of Chemistry, Loughborough University, Loughborough LE11 3TU, UK; \\ J.S.Sagu@lboro.ac.uk (J.S.); U.Wijayantha@lboro.ac.uk (U.W.); G.W.Weaver@lboro.ac.uk (G.W.) \\ 2 School of Design and Creative Arts, Loughborough University, Loughborough LE11 3TU, UK; \\ D.J.Southee@lboro.ac.uk (D.S.) \\ * Correspondence: P.F.Serobaba-Soares-Luis@lboro.ac.uk \\ + Presented at the International Conference on the Challenges, Opportunities, Innovations and Applications \\ in Electronic Textiles (E-Textiles 2019), London, UK, 12 November 2019.
}

Published: 5 December 2019

\begin{abstract}
The mass commercialization of electronic textiles is currently hindered due to the lack of reliable manufacturing technologies of energy storing textiles. To incorporate energy storage as a part of a garment, it must be manufactured in a systematic manner, allowing the integration of multiple components of electronic textiles simultaneously. Aiming towards process automation, carbon-based screen-printing inks were formulated for textile supercapacitor applications, focusing on the effect of carbon additives in the electrochemical performance. Devices were assembled, and their electrochemical performance was assessed. The maximum areal power density obtained was $12 \mathrm{~mW} \mathrm{~cm}^{-2}$ with an areal energy density of $1.08 \times 10^{-3} \mathrm{mWh} \mathrm{cm}^{-2}$.
\end{abstract}

Keywords: e-textiles; printed textile supercapacitors; ink formulation; carbon materials

\section{Introduction}

Electronic textiles are garments fitted with sensors and actuators, able to detect different signals from the environment and act. Potential applications may include embedded health-monitoring devices, lightweight military gear, and wearable computer fabrics [1]. Nontextile energy storage devices do not provide comfort and ease of movement as compared with the properties of the clothing itself, limiting the wide-spread usage of smart textiles. Thus, it is paramount to develop electric energy storage components that can be fully integrated into electronic textiles. Supercapacitors are energy storage devices whose properties are encountered between those of batteries and capacitors. While the energy that can be stored in a supercapacitor is lower than in a battery, the power capability is much higher. These devices store energy electrostatically, without electrochemical reactions and phase changes as observed in batteries, resulting in a higher cyclability compared with that of batteries [2].

With the increasing interest in wearable electronic devices, various flexible supercapacitors were investigated [3]; however, few studies focusing on printed carbon textile supercapacitors can be found in the literature.

Herein, electrodes comprised of different carbon materials were screen-printed and their electrochemical performance evaluated to search for the combination that reported the best storing properties. Subsequently, printed textile supercapacitors were manufactured and their performance assessed. 


\section{Materials and Methods}

\subsection{Ink Formulation}

Activated carbon Norit DLC Supra 30 (Cabot Norit Netherlands, Klazienaveen, The Netherlands) was used as the main filler in the ink, and Ketjenblack EC-300J (AkzoNobel Functional Chemicals, Amersfoort, The Netherlands) as the conductive additive.

An ink was made by adding carbon materials to an acrylic resin. Carbon materials were added gradually and mixed by hand. Milling was carried out using an EXAKT50EC three-roll mill (EXAKT Advanced Technologies GmbH, Norderstedt, Germany), with a predefined back and front gap of $15 \mu \mathrm{m}$ at a speed of $100 \mathrm{rpm}$. The inks were allowed to relax overnight before printing.

\subsection{Printing Methodology}

To analyze the electrochemical performance of high-surface-area carbon inks, printing of $2.5 \times 5.0 \mathrm{~cm}^{2}$ was carried out on hard stainless steel (Hollinbrow Precision Products Ltd., Telford, UK).

For full devices, flexible silver ink (Gwent Electronic Materials, Pontypool, UK) was first printed on a breathable polyester microfiber (Pennine Outdoor, Lancaster, UK), followed by a layer of graphene ink (Gwent Electronic Materials, Pontypool, UK), finalized by a $5.0 \times 5.0 \mathrm{~cm}^{2}$ layer of high-surface-area carbon ink. Finally, a PVA- $\mathrm{H}_{2} \mathrm{SO}_{4}$ gel electrolyte was printed and vacuum-impregnated at room temperature. The screen selection and drying parameters are listed in Table 1.

Table 1. Summary of printing and drying settings.

\begin{tabular}{ccc}
\hline Ink & Screen Used & Drying Regime \\
\hline Silver & Stainless steel 230T & 10 min at $130^{\circ} \mathrm{C}$ \\
Conductive carbon & Stainless steel 230T & 60 min at $150{ }^{\circ} \mathrm{C}$ \\
Active layer & Monofilament polyester 32T & 30 min at $120^{\circ} \mathrm{C}$ \\
Gel electrolyte & Monofilament polyester 32T & Room temperature \\
\hline
\end{tabular}

\subsection{Electrochemical Characterization}

A three-electrode system was assembled by using the printed carbon electrode on stainless steel as the working electrode, a Pt-mesh as the counter, and a $1 \mathrm{M}$ silver chloride electrode as the reference. Cyclic voltammetry (CV), galvanostatic cycling (GC), and electrochemical impedance spectroscopy (EIS) were used to characterize the electrochemical properties of the electrode, performed with an Autolab PGSTAT12 potentiostat. CVs were performed between -0.2 and $0.6 \mathrm{~V}$ (vs. $1 \mathrm{M} \mathrm{Ag} / \mathrm{AgCl}$ ) in a $1 \mathrm{M} \mathrm{H}_{2} \mathrm{SO}_{4}$ electrolyte at a scan rate of $5 \mathrm{mV} \mathrm{s}^{-1}$. Galvanostatic cycling was carried at $0.5 \mathrm{~mA} \mathrm{~cm} \mathrm{~cm}^{-2}$. The electrochemical impedance spectra were carried out at open-circuit potential over a frequency range of 0.01 to $100,000 \mathrm{~Hz}$ with $10 \mathrm{mV}$ amplitude.

As for the devices, the performance was evaluated through CV, GC, and EIS. CVs were performed between 0 and $0.8 \mathrm{~V}$ at scan rates ranging between 5 and $300 \mathrm{mV} \mathrm{s}^{-1}$. Galvanostatic cycling was carried out between 0.4 and $1.6 \mathrm{~mA} \mathrm{~cm}^{-2}$. The electrochemical impedance spectroscopy was carried out over a frequency range of 0.01 to $100,000 \mathrm{~Hz}$ with a $10 \mathrm{mV}$ amplitude.

\section{Results and Discussion}

\subsection{Electrochemical Performance of Printed Active Layers}

The amorphous nature of activated carbon, allied with its rough surface, leads to poor electrode electrical conductivity. Although activated carbon is the main component of a supercapacitor electrode due to their inherent high surface area, conductive additives are required to decrease the interparticle electrical resistance, thus increasing the overall electrode electrical conductivity. Carbon blacks are commonly employed in relatively low concentrations to compensate for the lack of electrical conductivity of the activated carbon, meaning that a low dosage is required to achieve the percolation threshold [4]. 
To demonstrate the effect of conductive additives, printed electrodes comprised of activated carbon (AC) and a blend of activated carbon and carbon black (AC:KB300) were subjected to a series of electrochemical analyses, and the results are reported in Figure 1. Printed carbon electrodes of AC and AC:KB300 have a mass loading of 16.5 and $12.7 \mathrm{mg} \mathrm{cm}^{-2}$, respectively.

Figure 1a shows cyclic voltammograms at $5 \mathrm{mV} \mathrm{s}^{-1}$; the typical rectangular signal is observed in both printed electrodes and no redox peaks were detected, suggesting that no redox-active species were present in the printed electrodes. It should be noted that the CVs of printed electrodes of AC:KB300 exhibit a larger area compared with that of AC, demonstrating higher storing capabilities.

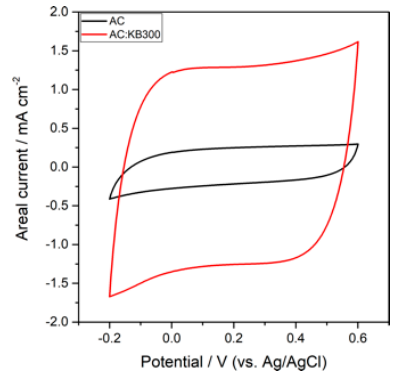

(a)

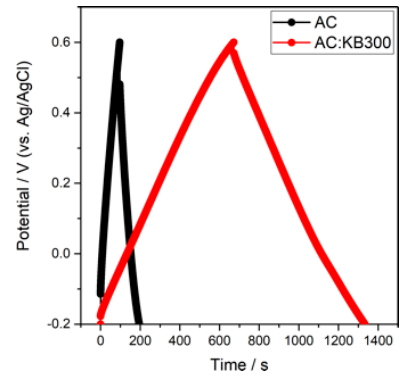

(b)

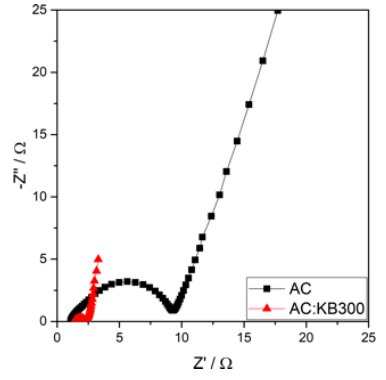

(c)

Figure 1. Electrochemical performance of high-surface-area carbon electrodes: (a) Cyclic voltammetry at a scan rate of $5 \mathrm{mV} \mathrm{s}^{-1} ;(\mathbf{b})$ galvanostatic cycling at $0.4 \mathrm{~mA} \mathrm{~cm}^{-2} ;$ (c) Nyquist plot.

Galvanostatic cycling at $0.4 \mathrm{~mA} \mathrm{~cm}-2$ of the printed electrodes is reported in Figure $1 \mathrm{~b}$. As observed, printed layers of AC:KB300 show higher discharge periods than those of printed layers of AC, which translates to higher areal capacitances of 330.6 and $49.1 \mathrm{mF} \mathrm{cm}^{-2}$, respectively. Moreover, the internal resistance of both printed active layers is 6.1 and $23.7 \Omega$. The reported enhancement of the storing and rate capability of printed electrodes evidences the importance of conductive additives in the performance of supercapacitors.

Electrochemical impedance spectra of both printed active layers is reported in Figure 1c. The results support the previous claim that the addition of carbon blacks decreases the resistance of the electrode. This is noted by the differences in size between the spectra, whereas the printed layer with the conductive additive shows lower values of impedance.

\subsection{Electrochemical Performance of the Device}

Silver possesses high electrical conductivities and chemical inertness to ambient conditions when compared with other metals [5]. However, it has been dismissed as a current collector material for printed supercapacitors due to electrolyte degradation occurring when in contact with waterbased electrolytes, damaging the performance of the device [6]. Herein, an interlayer comprised of graphene ink was added to cease the physical contact of the electrolyte while maintaining the conductive pathways between the printed active layer and the highly conductive current collector.

Full devices were manufactured and characterized in order to gain information regarding the storing characteristics. An illustration of the device can be seen in Figure 2a. As depicted, two printed textile electrodes, both comprised of a printed silver layer followed by a printed graphene layer and a printed active layer, were sandwiched. Cyclic voltammograms at different scan rates are shown in Figure $2 \mathrm{~b}$. At low scan rate the $\mathrm{CVs}$ demonstrate a rectangular shape. With increasing scan rates, the skewing of the signal is observed, suggesting that the active surface is highly porous, thus hindering the storing process at the electrode-electrolyte interface.

The galvanostatic cycling of the device is reported in Figure 2c. The device was cycled between 0.4 and $1.6 \mathrm{~mA} \mathrm{~cm}^{-2}$, demonstrating an areal capacitance of 57.0 and $16.5 \mathrm{mF} \mathrm{cm}^{-2}$, respectively. Moreover, the internal resistance of the device was $16.5 \Omega$. 


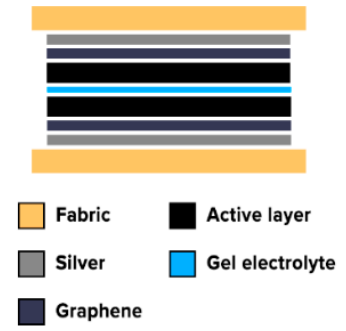

(a)

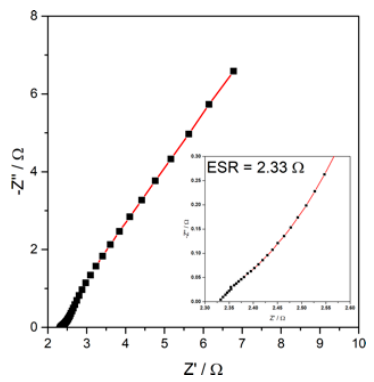

(d)

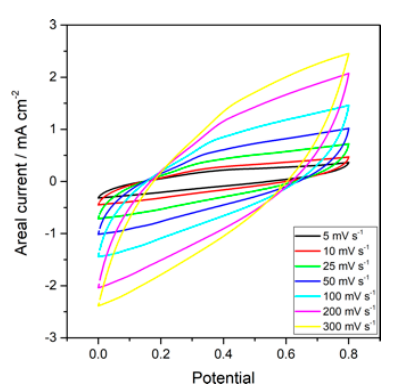

(b)

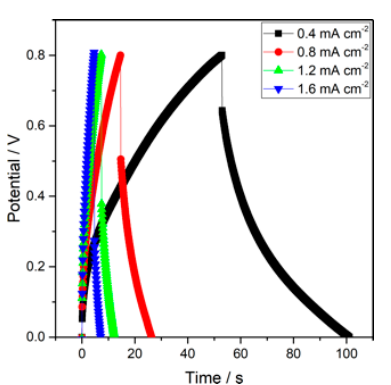

(c)

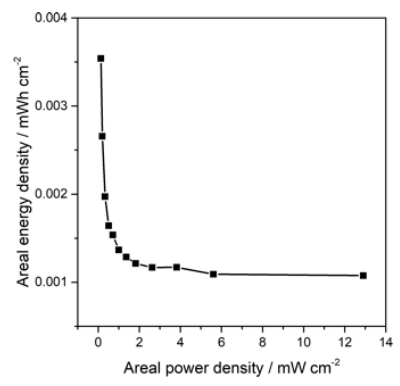

(e)

Figure 2. Electrochemical performance of full device: (a) Schematic of the device; (b) cyclic voltammetry; (c) galvanostatic cycling; (d) Nyquist plot; (e) Ragone plot.

The representation of the electrochemical impedance spectrum in a Nyquist plot is shown in Figure 2d. At high frequencies, the ESR of the device can be extracted, and the value obtained for the device was $2.3 \Omega$. At lower frequencies, a rather capacitive process occurs, which translates into a higher accessibility of the ions present in the electrolyte that contribute to the storing of energy at the electrode-electrolyte interface. Given the shift of the signal towards $45^{\circ}$ at low frequencies, it can be stated that the active layer possesses a high density of pores, meaning that the device is not fully charged. Moreover, this behavior could be intensified due to the viscous nature of the gel electrolyte, which decreases the mobility of the ions. A Ragone plot of the device can be seen in Figure 2e. At low current densities, a maximum areal energy density of $3.54 \mu \mathrm{Wh} \mathrm{cm}^{-2}$ was obtained, whereas at high

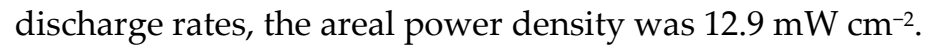

\section{Conclusions and Future Work}

High-surface-area carbon inks were developed, aiming towards the printing of textile supercapacitors to power e-textiles. The presence of carbon additives demonstrated an enhancement in the electrochemical performance, which was best shown in inks with carbon black, obtaining $330.6 \mathrm{mF} \mathrm{cm}^{-2}$ in a three-electrode setup. A full device was assembled and tested. An areal capacitance of $57.0 \mathrm{mF} \mathrm{cm}^{-2}$ was obtained at low currents, corresponding to a maximum areal energy density of $3.54 \mu \mathrm{Wh} \mathrm{cm}{ }^{-2}$. At high discharge rates, the areal power density was $12.9 \mathrm{~mW} \mathrm{~cm}{ }^{-2}$. Future research will be conducted in methodologies to increase the electrolyte penetration through the inner porous structure of the electrode, thus increasing the storing capabilities of the device.

Funding: The EPSRC are thanked for funding the Doctoral Training Partnership (LU, UK). U.W. thanks EPSRC for the support of the Joint University Industry Consortium for Energy materials and Devices (JUICED) hub (EP/R023662/1).

Conflicts of Interest: The authors declare no conflict of interest.

\section{References}

1. Stoppa, M.; Chiolerio, A. Wearable Electronics and Smart Textiles: A Critical Review Sensors 2014, 14, 11957-11992.

2. Eftekhari, A. The mechanism of ultrafast supercapacitors. J. Mat. Chem. A 2018, 6, 2866-2876. 
3. Qi, X.; Jinfeng, S.; Yan, H.; Minshen, Z.; Zengxia, P.; Hongfei, L.; Yukun, W.; Na, L.; Haiyan, Z.; Chunyi, Z.; Recent Progress on Flexible and Wearable Supercapacitors. Small 2017, 13, 1701827.

4. Jäckel, N.; Weingarth, D.; Schreiber, A.; Krüner, B.; Zeiger, M.; Tolosa, A.; Aslan, M.; Presser, V. Performance evaluation of conductive additives for activated carbon electrolytes in organic electrolyte. Electrochim. Acta 2016, 191, 284-298.

5. Venkata, K.R.R.; Venkata, A.K.; Karthik, P.S.; Surya, P.S. Conductive silver inks and their applications in printed and flexible electronics. RSC Adv. 2015, 5, 77760.

6. Jagdeep, S.S.; Nicola, Y.; Darren, S.; Upul, K.G.W.; Printed electrodes for flexible, light-weight solid-state supercapacitors-A feasibility study. Circuit World 2015, 41, 80-86.

(C) 2019 by the authors. Licensee MDPI, Basel, Switzerland. This article is an open access article distributed under the terms and conditions of the Creative Commons Attribution (CC BY) license (http://creativecommons.org/licenses/by/4.0/). 